\title{
Partial reversal of protan and tritan colour defects with inhaled oxygen in insulin dependent diabetic subjects
}

Fiona M Dean, Geoffrey B Arden, Anne Dornhorst

\begin{abstract}
Aims-Abnormalities in colour perception occur early in the development of diabetic retinopathy. Whether these changes can be influenced by increasing circulating oxygen saturation was studied in comparison with non-diabetic controls. Methods-Protan and tritan colour thresholds were measured using a computer graphics system in 37 insulin dependent diabetic subjects, with no or minimal background retinopathy, and 27 matched controls. Colour thresholds were performed after subjects inhaled either gaseous air or $100 \%$ oxygen for a minimum of 5 minutes.
\end{abstract}

Results-Diabetic subjects had higher colour vision thresholds when inhaling air when compared with controls (protan (mean 3.93 (SEM 0.39), $v 2.36$ (0.16), $\mathrm{p}<0.0002)$ and tritan $(8.15(0.62) \quad v \quad 5.42$ $(0.31), p<0.002))$. The colour vision thresholds observed in diabetic subjects inhaling air fell when they inhaled oxygen (protan (3.93 (0.39) $v 3.57(0.33), \mathrm{p}<0.025)$ and tritan $(8.15 \quad(0.62) \quad v \quad 7.35 \quad(0.59)$, $p<0.005)$ ). No fall in colour thresholds was seen in non-diabetic controls who inhaled oxygen.

Conclusion-A small improvement in the colour vision thresholds was observed using computer graphics in diabetic subjects, with minimal or no retinopathy, who inhaled oxygen. This study supports a hypothesis that reduced retinal oxygenation contributes to the colour vision defects in diabetes.

(Br F Ophthalmol 1997;81:27-30)

Moorfields Eye

Hospital, Institute of

Ophthalmology,

London EC1 V2PD

F M Dean

G B Arden

Department of Medicine, University

College London

Medical School,

Whittington Hospital,

London N19 3UA

A Dornhorst

Correspondence to:

Fiona $M$ Dean

Eye Department,

St Thomas's Hospital,

London SE1.

Accepted for publication 14 October 1996 vision, perhaps as a consequence of relativ retinal oxygen desaturation. ${ }^{4}$ We examined, using computer graphics technology, ${ }^{5-7}$ whether increasing circulating oxygen saturation improved the raised colour vision thresholds in diabetic subjects.

\section{Materials and methods}

Thirty seven, young, normotensive, insulin dependent diabetic (IDDM) patients were recruited from an inner city diabetic outpatient clinic. Assessment of diabetic control was obtained from their last outpatient $\mathrm{HbA}_{1 \mathrm{c}}$ value. All subjects had no or minimal diabetic retinopathy ( $\leq$ grade 1$).^{8}$ No patient had received laser therapy, had previous ocular disease, or treatment likely to affect colour vision. Twenty seven non-diabetic hospital personnel of similar age without ocular pathology were recruited as controls. Patients and controls gave informed consent, the study having been previously approved by the hospital's medical ethics committee.

Studies were performed in the hospital's ophthalmology department. Corrected visual acuity was measured using the Snellen chart. The eye with the better visual acuity was tested for colour contrast sensitivity with the other eye occluded.

Colour contrast sensitivity was assessed measuring protan and tritan thresholds using a computer graphics system as previously described..$^{5-7}$ In order for the alphabetical letter displayed on the computer screen to subtend a constant angle on the retina, the subject is seated at a fixed distance from the screen. The size of the letter is set to create an image that tests the central 6 degrees of the retina. The letters displayed on the screen are of variable intensity on a background of equiluminance. The operator has no influence on the contrast of the test letter given and could only influence the result by indicating a wrong response. The computer finds the endpoint of the test by a double staircase method; this offers a darker letter that is easier to see following a wrong answer and a lighter letter following a correct answer. The difference in the contrast between successive jumps was $50 \%$ of the previous step. This process inevitably leads to finite steps which reach a plateau at the colour contrast sensitivity threshold. The reproducibility of this measurement is $3 \%$, which is taken as the sensitivity of the test.

After an initial learning period when colour thresholds were assessed with the subject breathing room air without a face mask, the test was repeated twice more with the subject inhaling either bottled air ( $21 \%$ oxygen) or bottled oxygen $(100 \%)$ through a $60 \%$ Venti- 
Table 1 Characteristics of 37 insulin dependent diabetic subjects and 27 controls

\begin{tabular}{lll}
\hline & Diabetic & Controls \\
\hline Male:female & $21: 16$ & $11: 16$ \\
Age (SE) years & $35(1)$ & $31(2)$ \\
$\begin{array}{l}\text { Duration of diabetes } \\
\text { Median (range) years }\end{array}$ & $11(1-35)$ & - \\
White:non-white & $32: 5$ & $22: 5$ \\
\hline
\end{tabular}

mask. The gases were administered for $5 \mathrm{~min}$ utes before threshold sensitivities were measured. Although the person giving the test knew which gas was being used, the subject did not, and the order of the inhaled gases was alternated between subjects to minimise any learning effect that might bias the results.

Dilated funduscopy was performed by the same observer (FMD) in all subjects. The presence or absence of diabetic retinopathy was graded according to the modified Airlie House classification. ${ }^{8}$

Changes in oxygen saturation in the peripheral circulation were measured using digital pulse oximetry (Satelite, manufactured by Datex) in 19 subjects (13 diabetic and six control subjects) while inhaling gaseous air and $100 \%$ oxygen.

Control subjects were selected to be of similar age and ethnic mix as the diabetic subjects. However there was a higher male:female representation in the IDDM subjects (Table 1 ). The patient group had a median duration of diabetes of 11 years (range 1-35, mean 13.0). Diabetic control at the time of the study was good, mean $\mathrm{HbA}_{1 \mathrm{c}} 7.4 \%$ (range 5.6-11.9, non-diabetic range $4-6.5 \%$ ). In addition to insulin, one diabetic patient was receiving treatment for both ocular myasthenia (pyridostigmine) and hyperthyroidism (carbimazole), being well controlled for both conditions.

Normally distributed data were expressed as mean (SEM), with skewed data (when the arithmetical mean and geometric mean were dissimilar) expressed as median and range. Statistical comparisons between the groups was made using an unpaired Student's $t$ test and $\chi^{2}$ tests with continuity correction analysis as appropriate. Individual comparisons within the groups were analysed using the paired Student's $t$ test. Correlations were performed using simple regression analysis. Significance was defined as $\mathrm{p}<0.05$.

\section{Results}

No diabetic or control subject had a corrected visual acuity of $<6 / 18$. Corrected visual acuity in 25/36 of the diabetic and 23/26 of the control groups was $\geq 6 / 5$. Eighteen of the 37
IDDM subjects had no background diabetic retinopathy, while 19 IDDM subjects had background retinopathy (grade 1).

The assessment of colour contrast sensitivity thresholds using the computer graphics system was well tolerated by all subjects, the only discomfort expressed being short lived visual artefacts caused by uniocular occlusion. A learning effect, as anticipated, was observed in both the diabetic and control subjects. The initial tritan and protan thresholds obtained breathing room air were consistently higher than those obtained subsequently breathing gaseous air via a face mask (Table 2).

The arterial oxygen saturation percentage in the peripheral circulation in the diabetic and control subjects was similar when inhaling gaseous air (97.8 (SEM 0.90) v 98.2 (0.75), respectively) and rose in both groups with inhaled oxygen (99.6 (0.24) $v 100.00(0.01)$, respectively).

Protan thresholds were significantly higher in the diabetic than the control subjects inhaling both gaseous air (3.93 (0.39) v 2.36 (0.16), $\mathrm{p}<0.0002)$ and oxygen $(3.57(0.33) v 2.49$ (0.17), p <0.02), (Fig 1). The diabetic subjects, but not the controls, had significantly lower protan thresholds when inhaling oxygen compared with air (3.57 (0.33) v $3.93(0.39)$, p $<0.025$ ), with a $>3 \%$ decrease in threshold sensitivity being observed in 24/37 of the diabetic individuals as opposed to only $5 / 27$ of the controls $\left(\chi^{2}=11.73, \mathrm{p}<0.025\right)$.

Tritan thresholds were significantly higher in the diabetic than the control subjects when inhaling gaseous air (8.15 (0.62) v $5.42(0.31)$, $\mathrm{p}<0.002)$ or oxygen $(7.35(0.59) v 5.46$ (0.30), p <0.05), (Fig 1). Diabetic subjects had significantly lower tritan thresholds when inhaling oxygen compared with air (7.35 (0.59) $v 8.15$ (0.62), $\mathrm{p}<0.005)$, with a $>3 \%$ decrease in thresholds being observed in $21 / 37$ of the diabetic individuals compared with $7 / 27$ of the controls $\left(\chi^{2}=5.23, \mathrm{p}<0.05\right)$.

There was a significant, positive correlation between the duration of diabetes and colour sensitivity thresholds for protan and tritan when inhaling either air $\left(r^{2}=0.129,95 \%\right.$ confidence limits (CL) 3.08-4.57, p $<0.05$ and $r^{2}=$ $0.289,95 \%$ CL $0.1-0.33, \mathrm{p}<0.001$, respectively) or oxygen $\left(r^{2}=0.121,95 \% \mathrm{CL}\right.$ $0.004-0.14, \mathrm{p}<0.05$ and $r^{2}=0.356,95 \% \mathrm{CL}$ $0.84-2.32, \mathrm{p}<0.0001$, respectively).

The protan and tritan colour thresholds in the 18 diabetic subjects with no retinopathy (grade 0) were significantly lower than the thresholds of the 19 subjects with grade 1 retinopathy (protan $(2.72(0.21) v 5.07(0.63))$ and tritan (6.2 1(0.46) v $10.0(0.95)), \mathrm{p}<0.002$

Table 2 Protan and tritan colour contrast sensitivity percentage thresholds in 37 insulin dependent diabetic subjects and 27 controls during an initial learning test run breathing room air and during the study inhaling gaseous air via a Ventimask

\begin{tabular}{llllll}
\hline & \multicolumn{3}{l}{ Diabetic subjects } & & \multicolumn{2}{c}{ Control subjects } \\
\cline { 2 - 3 } & Room air test run & $\begin{array}{l}\text { Gaseous air } \\
\text { Ventimask }\end{array}$ & & Room air test run & $\begin{array}{l}\text { Gaseous air } \\
\text { Ventimask }\end{array}$ \\
\hline Protan sensitivity \% thresholds & $4.44(0.41)$ & $3.93(0.39)^{\star}$ & & $3.08(0.22)$ & $2.36(0.16) \dagger$ \\
Tritan sensitivity \% thresholds & $8.74(0.74)$ & $8.15(0.62)$ & & $6.07(0.34)$ & $5.42(0.31) \dagger$ \\
\hline
\end{tabular}

*Significant difference between test run on room air and gaseous air via Ventimask in diabetic subjects, $\mathrm{p}<0.05$.

†Significant difference between test run on room air and gaseous air via Ventimask in control subjects, $\mathrm{p}<0.05$. 


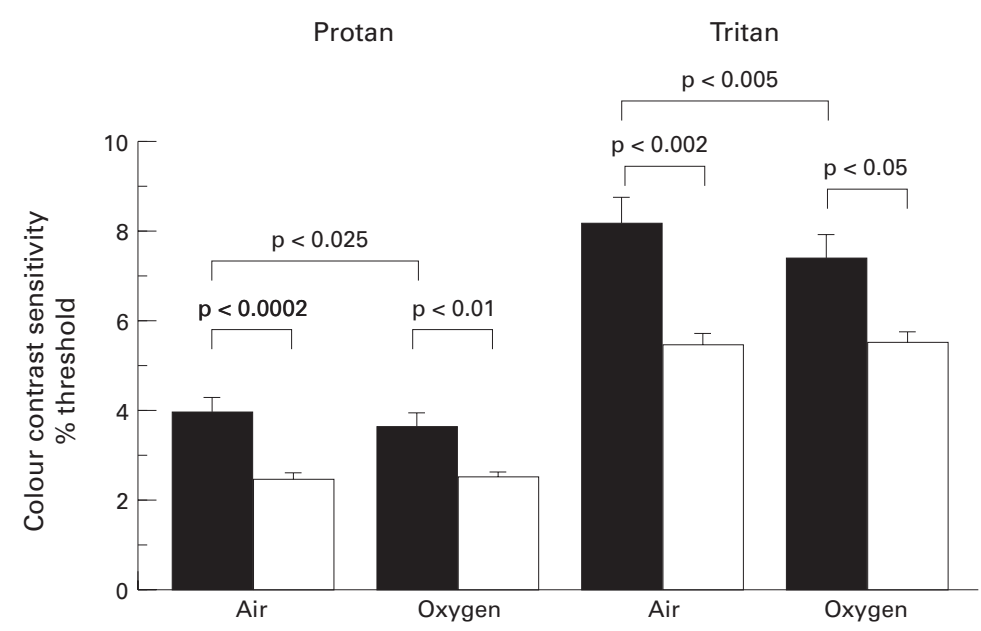

Figure 1 Protan and tritan colour contrast sensitivity percentage thresholds in 37 insulin dependent diabetic subjects (solid bars) and 27 non-diabetic controls (open bars) when inhaling gaseous air and 100\% oxygen.
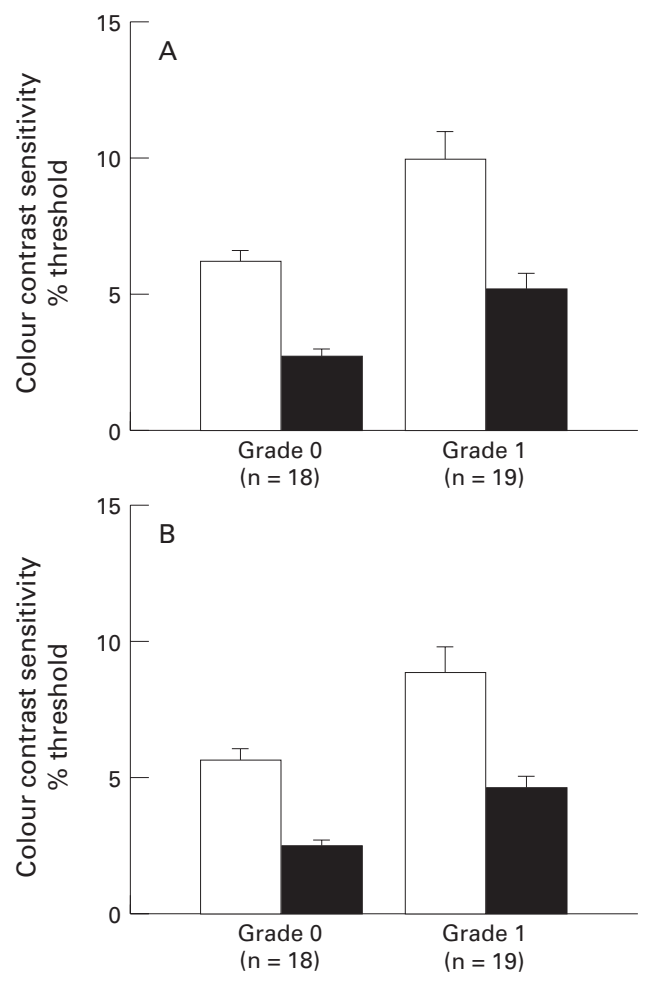

Figure 2 Protan and tritan colour contrast sensitivity percentage thresholds in 37 insulin dependent diabetic subjects according to grade ( 0 or 1 ) of diabetic retinopathy when inhaling gaseous air $(A)$ and $100 \%$ oxygen $(B)$.

in both instances). The response of colour thresholds to inhaled oxygen was of a similar magnitude in diabetic subjects with and without retinopathy (Fig 2).

\section{Discussion}

This study confirms abnormalities of both protan and tritan colour vision in diabetic subjects with normal visual acuity and no or minimal background retinopathy. ${ }^{2}$ These differences in colour thresholds could not be explained by age. ${ }^{9}$ Protan and tritan vision thresholds increased with the duration of diabetes and were higher in the diabetic subjects with grade 1 retinopathy than those with none. Our study shows for the first time that the increased colour contrast sensitivity thresholds associated with diabetes can be lowered with inhaled oxygen.

Optimal colour perception is likely to be dependent on high retinal oxygenation. Defects in colour vision occur in non-diabetic subjects at altitude when arterial oxygen saturation is low and correct when the oxygen saturation is raised. ${ }^{10}$ Abnormalities of colour perception in diabetic subjects could result from either compromised retinal arterial oxygen saturation or reduced oxygen tension in the tissue. Small increases in oxygen saturation of the peripheral circulation, similar to those achieved in this study (97 to $100 \%$ ), would be expected to facilitate oxygen exchange owing to the kinetics of the oxygen-haemoglobin dissociation curve at high saturation. While the oxygen saturation of the peripheral circulation will not, necessarily, reflect the saturation of the retinal circulation, it is likely that the retinal oxygen saturation would also rise in response to inhaled oxygen.

Normal subjects have a vasoconstrictive response in the retina to inhaled oxygen, a response known to be lost early in diabetes. ${ }^{112}$ Changes in retinal circulation occur in diabetes with areas of hyperperfusion due to capillary shunting and hypoperfusion due to in situ microthrombi, the latter contributing to retinal ischaemia. ${ }^{13}$ It is possible that abnormal colour vision in diabetic subjects arises from retinal photoreceptors functioning at suboptimal levels of oxygenation. If low retinal oxygenation is a causal factor in the raised colour contrast sensitivity thresholds found in diabetes, improvement of colour vision by increasing retinal oxygenation is to be predicted. Conversely, in healthy subjects with normal retinal oxygenation and optimal cone function further improvement in colour vision would not be expected with inhaled oxygen. Theoretically, a rise in colour contrast sensitivity thresholds could occur as a result of retinal vasoconstriction.

The differences in tritan and protan colour contrast sensitivity thresholds between the diabetic and control subjects observed with and without inhaled oxygen are unlikely to have been explained by a learning effect. The purpose of the initial learning run was to adjust for any bias which might be the result of a learning effect. Alternating the test order of the inhaled gases would have further reduced any effect of learning bias on the results. Theoretically, the slightly greater male predominance in the diabetic than the control group could have influenced the results on protan but not tritan vision. The small differences in visual acuity between the two groups would not be expected to influence colour perception .

We believe that this study provides new evidence that reduced retinal oxygenation may be a causative factor for colour visual defects in diabetic subjects with no or minimal diabetic retinopathy. Further work is needed to establish whether this finding applies to diabetic subjects with more advanced grades of retinopathy. 
FMD was funded in part by a grant from the locally organised research scheme at Moorfields Eye Hospital.

1 Treagear SD, Knowles PJ, De Alwys DV, Reffin JP, Ripley LG, Casswell AG. Colour vision deficits predict the development of sight-threatening disease with background
retinopathy. Invest Ophthalmol Vis Sci 1993;34 (ARVO retinopathy. Invest $O$ Pht
Abstracts no 81):719.

2 De Alwys DV, Reffin JP, Tregear SJ, Ripley LG, Casswell AG. Should the management of diabetic retinopathy be based upon measurements of visual function rather than observations of retinal morphology? Invest Ophthalmol Vis Sci 1993;34 (ARVO Abstracts no 81):719.

3 Hardy KJ, Lipton J, Scase MO, Foster DH, Scarpello JHP. Detection of colour vision abnormalities in uncomplicated type 1 diabetic patients with angiographically normal retinas. Br f Ophthalmol 1992;76:461-4.

4 Kawagishi T, Nishzawa Y, Emoto M, Konishi T, Maekawa $\mathrm{K}$, Hagiwara S, et al. Impaired retinal artery blood flow in IDDM patients before clinical manifestations of diabetic IDDM patients before clinical manifestati

5 Arden GB. Testing contrast sensitivity in clinical practice. Arden GB. Testing contrast
Clin Vis Sci 1987;2:213-24.
6 Arden GB, Gunduz K, Perry S. Colour vision testing with a Arden GB, Gunduz K, Perry S. Colour vision testing with
computer graphics system. Clin Vis Sci 1988;2:303-20.

7 Arden GB, Gunduz K, Perry S. Colour vision testing with a Arden GB, Gunduz K, Perry S. Colour vision testing with a
computer-graphics system; preliminary results. Doc Ophhalmol 1988; 69:167-74.

8 Diabetic Retinopathy Research Group. A modification of the Airlie House classification of diabetic retinopathy. Invest Ophthalmol Vis Sci 1981;21 (Diabetic Retinopathy Study Report no 10):210-26.

9 Ruddock KH. The effect of age on colour vision. 1: response in the receptoral system of the eye. Vis Res 1965;5:37-45.

10 Richalet JP, Rutgers V, Bouchet P, Rymer JC, Keromes A Duval-Amould G, et al. Diurnal variations of acute mountain sickness, colour vision, and plasma cortisol and ACTH at altitude. Aviat Space Environ Med 1989;60:105-11.

11 Hickan JB, Sieker HO. Retinal vascular reactivity in patients with diabetes mellitus and with atherosclerosis. Circulation 1960;22:240-3.

12 Grunwald JE, Riva CE, Brucker AJ, Sinclair SH, Petrig BI. Altered retinal vascular responses to $100 \%$ oxygen breathing in diabetes mellitus. Ophthalmology 1984;91:1447-52.

13 Davies MD, Rand L. Diabetic retinopathy. In: Alberti KGMM, DeFronzo RA, Keen H, Zimmet P, eds. International textbook of diabetes mellitus. Chichester: John Wiley, 1992. 